\title{
Challenges in delivering rheumatology care during COVID-19 pandemic
}

\author{
A. Nune ${ }^{1}$ (D) K. Iyengar ${ }^{1}(D) \cdot A$. Ahmed $^{1} \cdot$ H. Sapkota ${ }^{2}$ \\ Received: 29 June 2020 / Revised: 22 July 2020 / Accepted: 23 July 2020 / Published online: 25 July 2020 \\ (C) International League of Associations for Rheumatology (ILAR) 2020
}

\begin{abstract}
COVID-19 has significantly affected healthcare systems around the world. To prepare for this unprecedented emergency, elective patient care was put on hold across the National Health Service (NHS). Rheumatology service had to be reorganised with a cancellation of elective clinics and clinical reconfiguration to continue to deliver care to patients, support frontline, and prevent viral transmission. The rheumatology community's responsibility of providing a continuity of care for patients had to be balanced with measures to reduce the risk of viral transmission and also protection of both the patients and staff. We describe our experience of delivering rheumatology service as recommended by the National Institute for Health and Care Excellence (NICE NG167) guidelines at a district general hospital during the current pandemic.

Key Points

- Prepare to deliver a rapid mass communication; ensure email and mobile phones registered in patients' records; enable access to text and video messaging.

- To ensure wider access to innovative digital technology in clinical practice; implement telephone and video consultations where appropriate.

- To consider setting up community OP clinics, for example, mobile and satellite clinics.
\end{abstract}

Keywords COVID-19 · Coronavirus · Pandemics $\cdot$ Rheumatology $\cdot$ Telemedicine

\section{Introduction}

The novel coronavirus SARS-CoV-2 pandemic began in Wuhan (China) in December 2019 and has spread worldwide [1]. It is a highly contagious infection with predominantly respiratory symptoms which could be life threatening [2, 3]. Centers for Disease Control and Prevention (CDC) has

\section{A. Nune}

arvind.nune@nhs.net

\section{K. Iyengar}

kiyengar@nhs.net

A. Ahmed

Ashar.Ahmed1@nhs.net

H. Sapkota

hem.sapkota@nhs.net

Southport and Ormskirk NHS Trust, Southport, UK

2 The Royal Wolverhampton NHS Trust, Wolverhampton Road, Wolverhampton, UK published guidelines to avoid contracting the illness and prevent its spread [4]. To deliver safe and effective care, the British Society for Rheumatology (BSR) and National Institute for Health and Care Excellence (NICE) have produced guidelines for the management of patients with rheumatological autoimmune inflammatory and metabolic bone disorders during the current pandemic (Table 1) [5, 6]. The rheumatology community had to achieve a balance of protecting the patients and staff, reducing the risk of spread of viral transmission whilst providing a safe continuous clinical care.

\section{Patient communication and risk management}

Following NICE and BSR guidance in early April 2020, our cohort of patients was risk categorised into one of the three risk groups - risk group 1 (mild), 2 (moderate) and 3(severe) - depending on the immunosuppressive drugs patients were taking and their co-morbidities and was advised to follow guidelines on social distancing, self-isolation and shielding accordingly [7, 8]. Identifying at-risk patients and 
rapid mass communication was a huge challenge. There were many hurdles to identify patients who were taking synthetic disease-modifying antirheumatic drugs (DMARDs). The department had information for patients who had been started on DMARDs in the last 12 months. An updated list of patients on biologic drugs was obtained from the funding database of the hospital pharmacy. The department promptly sent advice to all 393 patients on biologics via post, which included a risk stratification chart so that patients could self-calculate their score or contact the department if they had any queries. Redeployment of the staff, sickness and with some in selfisolation meant that this was the only feasible option for the department to disseminate the guidance on time. We have contacted the first 100 patients to evaluate if the postal communication has been delivered. Ninety-two percent of the patients confirmed that they had indeed received the letters, and one patient had died which was unrelated to COVID-19. The patients who had received the guidance suggested that they have understood the information fully and either isolated or shielded accordingly. It is reassuring that patients did follow the guidance appropriate to their risk. Some of the patients who have not received the correspondence did contact the rheumatology helpline for clarification.

\section{Risk assessment of COVID-19 with modification of usual care}

The rheumatology department is led by two consultants, one middle-tier doctor and supported by specialist rheumatology nurses. Redeployment of medical staff to the COVID-19 frontline from a small department made the situation no easier. However, they were always available either on a phone or email to address any concerns. Some of our nurse specialists were in 'self-isolation' due to their medical history but worked from home to ensure continuity of care for the patients. Rheumatology helpline set-up was inundated with patient's queries mostly around COVID-19 which was carefully manned and appropriate advice given.

Patients were advised not to attend a face-to-face appointment when patients exhibited any symptoms for suspected COVID-19 such as cough, fever, or difficulty in breathing. All patients booked into the face-to-face clinics were asked screening questions for COVID-19. Fortunately, none of our patients who arrived for the face-to-face consultation showed any symptoms for COVID-19. Patients were encouraged not to arrive early for their appointments to reduce virus transmission. 'Social distancing' measures were in place in the outpatient seating areas. Recording routine observations such as weight and blood pressure were discouraged unless clinically needed, to avoid one more surface contact. Patients were advised to attend pre-booked radiology appointments to minimise the risk of virus transmission with advice regarding infection control. Patients were given access to the mask and hand sanitiser during their visit. Doctors and nursing staff ensured personal protective equipment (PPE) was worn at all times. A focused clinical examination was carried out where needed. Despite the negative media reports, PPE was available to use all times during the face-to-face encounters with the patients in our trust.
Table 1 Summary of adopted recommendations from NICE NG 167 and its implications on rheumatology clinical practice

\begin{tabular}{lll}
\hline \multicolumn{2}{c}{ Recommendations } & Service reconfiguration \\
\hline 1 & Patient communication and risk management & Mass communication by post \\
& & COVID-19 risk stratification advice \\
& Patient support by helpline \\
& Risk assessment with modification of usual care & COVID-19 screening and risk assessment \\
& Redeployment of consultants \\
& Safety/PPE \\
& Essential investigations only \\
& Outpatient clinic set-up & Patient clinic lists 'triaged and reorganised' \\
& Reduced face to face appointments \\
& Implemented remote consultations, e.g. telephone \\
& Treatment considerations & Continued DMARDs and biologic drugs \\
& Moved day care unit to community hospital \\
& Switched IV biologics to SC forms \\
& Intra articular injections on demand \\
& Delayed blood tests where possible \\
& Drug monitoring & Information/support \\
& BSR website guidance
\end{tabular}

NICE, National Institute for Health and Care Excellence; BSR, British Society of Rheumatology; DMARD, disease-modifying drugs; PPE, personal protective equipment; SC, sub-cutaneous; IV, intravenous 


\section{Out-patient clinic prioritisation}

Out-patient (OP) nursing appointments were converted to telephone clinics, but the consultant-led clinics were deferred to allow redeployment and provide cover to COVID-19 wards. Such patients from cancelled clinics underwent 'risk stratification' based on clinical information obtained from electronic patient records (EPR), medway patient administration system (PAS), haematology portal and picture archiving and communication system (PACS). New referrals with suspected early inflammatory arthritis, giant cell arteritis, connective tissue disease and small-vessel vasculitis were triaged for 'face-to-face' consultations where needed, and the rest of the patients were given telephone clinic appointments. Any urgent referrals identified from the list were seen during the weekend which allowed consultants to continue to care for COVID-19 in-patients during the week. This flexibility ensured continuity of the rheumatology service whilst providing COVID-19 emergency care at the frontline. Telephone consultations were arranged for routine follow-up patients with two reduced slots. The total number of patients seen per telephone clinic was restricted to 10 followups for the initial period, and each patient was given 20 -min slots to facilitate for any delay from technology glitches. Disease flare was managed with a short course of oral steroids during the peak of the pandemic. Patients were given a choice of receiving intramuscular corticosteroids where needed, but many patients opted for oral prednisolone.

\section{Treatment considerations}

There is little data available about the effect of novel SARSCoV-2 upon rheumatology patients with autoimmune conditions. However, knowing the potentially significant risk of disease flare-up if patient's immunosuppressive drugs were stopped, prompting concern of increased chance of hospital admissions during the pandemic, as a department, we decided to continue all immunosuppressive medications including biologic drugs [9]. To mitigate the risk of viral transmission, the day unit was moved to the nearby community hospital; therefore, all the biologic infusion drugs were continued as before unless the patient requested not to have it. All patients on intravenous (IV) infliximab, abatacept and tocilizumab for rheumatoid arthritis were given an opportunity to switch to subcutaneous (SC) forms as agreed locally. Out of the total of 16 patients on IV therapy, we successfully switched 7 patients to SC form. Although the benefits of switching from IV to $\mathrm{SC}$ administration was emphasised to our patients, 9 of them preferred not to switch due to various concerns which included 'needle phobia', fear of inefficacy with a newer form of drug and reluctance to switch during the middle of the pandemic. None of the patients was started on new biologic drugs during the pandemic, but after discussing the risks and benefits, some of the patients with new inflammatory arthritis were started on DMARDs.

Patients who were taking IV bisphosphonates such as zoledronic acid for osteoporosis were delayed; however, denosumab was continued. Routine injection clinics were stopped, but complementary measures were initiated to provide intra-articular injections on demand.

\section{Drug monitoring}

Following the updated NICE guidance, blood monitoring for DMARDs such as methotrexate was delayed for some patients based on clinical assessment once we had ensured it was safe to do so. For some, we commenced district nurses led support to help with blood monitoring of patients with overdue assessments, and thus, none were cancelled. Previous stable blood results and disease status, duration of therapy influenced these decisions. Unfortunately, a lot of blood monitoring still had to be done in the hospital as patients experienced difficulty getting their monitoring done through primary care.

\section{Discussion}

It was an enormous task to identify at-risk patients in our cohort and to communicate with them at speed. We had to rely on a traditional method of communication such as postal service which has its inherent drawbacks. Mass communication with novel methods such as video-embedded text messages, social media and email would have been a better option for speedy communication and assessment [10-12]. However, the lack of accessibility and set-up of these modes of communication were a limiting factor. An initiative in our trust was to speed up the implementation of 'Attend Anywhere' NHS (National Health Service) digital technology supported and promoted by the NHSE (National Health Service England) during the current pandemic [13]. 'Attend Anywhere', a web-based platform, has been centrally procured for all the healthcare trusts in England and will be available to use for 12 months. 'Attend Anywhere' platform provides a safe and secure link with the patients. However, there have been growing concerns about data security in other forms of video consultations such as Zoom. We anticipate that virtual consultations will be a routine part of the outpatient care post-COVID in the NHS. Video consultations would require patients to have access to the internet and either a computer or a mobile device which could potentially limit its wider use particularly in the elderly. To prepare for another emergency, we recommend an open discussion with patients and stakeholders to 
find the most suitable options to deliver communication safely and rapidly.

Lack of adequate staff due to sickness and redeployment was another important challenge to meet the current unprecedented demand. The effect must be similar in small departments like ours in the NHS and rheumatology departments across the world. The flood of helpline calls that the many rheumatology departments have probably experienced during the peak of the pandemic could be due to fear and anxiety in addition to their disease flare made worse by the barrage of conflicting information obtained from social media, press and even government sources [14]. Prompt delivery of consistent, clear, unambiguous and specific information directed to rheumatology patients using effective means of communication will reduce helpline queries to a manageable level. The effect of lack of staffing could be further mitigated to some extent by robust protocols and, more importantly, access to frequent virtual multidisciplinary team (MDT) meetings with the medical team via digital technology such as "Attend Anywhere" and WhatsApp portals. This also highlights the importance of providing remote Information and Communication Technology (ICT) access to staff to enable them to work remotely in case they are affected by pandemic directly or in self-isolation.

Strong decisive leadership with a consistent message helped run the department well during uncertain times. Our MDT meeting at the start of the pandemic did the same. We followed the national guidelines to manage our patients and provided a consistent management plan to nurse specialists. We continued biologic treatments to our entire patient cohort unless patients decided not to take them due to anxiety or fear of secondary infection. So far, there is no evidence of increasing incidence of COVID-19 cases in rheumatology patients receiving biologics which is reassuring [15].

The way we deliver rheumatology care probably would change in the near future, and we as a community need to adapt to the new ways of delivering care. There will be patients who will be apprehensive attending for 'face-to-face' consultations as elective services resume, and we need to ensure that the clinic environment is safe to deliver innovative care. Maintaining social distancing measures might be thought provoking once the OP clinics start running at full capacity, as in many places, rheumatologists share clinic areas with other specialities. The wider use of digital technology will assist in adapting to virtual clinics, but access to video consultations does have limitations, as some of its users may belong to an elderly group who may not be tech-savvy.

As we remember sacrifices the medical community has made during the pandemic, a greater emphasis to protect the frontline clinicians whilst continuing to provide safe and effective care for our patients is the need of the hour. As the COVID-19 antibody test becomes increasingly available, knowing antibody status will reassure both clinicians and patients and may restore their faith in the 'face-to-face' consultations. In the meantime, telemedicine revolution will support clinical services.

It was an immense challenge to adapt and manage rheumatology patients without the benefit of face-to-face consultations. Redeployment of the staff and self-isolation had significantly reduced our available skills on the ground. A wellestablished telephone helpline service in the department has helped to alleviate patients' anxiety and concerns about their health and well-being which ensured the continuity of care during the peak of the pandemic. Department was able to meet significantly increased service demands by quickly scaling up of our ICT access to the staff enabling them to work remotely. The department was able to use telemedicine effectively in multiple aspects of patient care including remotely monitoring disease activity, treatment changes, follow up and patient education. We believe that the wider world could learn from our initial shortcomings and adapt the ways of virtual consultations which will have a vital role in delivering rheumatology care during the pandemic. Any change in current practice needs to be monitored for improvement in delivering safe patient care and better patient satisfaction $[16,17]$.

\section{Conclusion}

The COVID-19 outbreak has thrown a new challenge to the unassuming rheumatology community which was already under stretch due to short staffing at the time of increasing demand. The experience gained during the pandemic has helped the rheumatology community to adapt to new ways to deliver OP rheumatology care and has shown resilience in the face of adversity. Although new COVID-19 cases are in a decline, non-face-to-face consultations will be here to stay. Telemedicine will be a key development in delivering future rheumatology patient care. The need for wider access to innovative digital methods to provide effective and safe patient care with remote consultations has become more relevant than ever before.

Authors' contributions $\mathrm{AN}$ and $\mathrm{KI}$ involved in conceptualization, literature search, manuscript writing and editing. AA involved in literature search, manuscript writing and references. HS supervised the overall submission and edited and approved the final draft. All authors read and agreed the final draft submitted.

\section{Compliance with ethical standards}

Conflict of interest The authors declare that they have no conflicts of interest.

Statement of ethics The current submitted article is not a clinical study and does not involve any patients. 


\section{References}

1. Huang C, Wang Y, Li X et al (2020) Clinical features of patients infected with 2019 novel coronavirus in Wuhan, China [published correction appears in lancet. Jan 30]. Lancet 395(10223):497-506. https://doi.org/10.1016/S0140-6736(20)30183-5

2. Du RH, Liang LR, Yang CQ et al (2020) Predictors of mortality for patients with COVID- 19 pneumonia caused by SARS-CoV-2: a prospective cohort study. Eur Respir J 55(5):2000524. Published 2020 may 7. https://doi.org/10.1183/13993003.00524-2020

3. WHO Dashboard Coronavirus (2020) Available from: https:// experience.arcgis.com/experience684d0521648f8a5beeeee1b9125cd. Accessed on 24 May 2020

4. Centers for Disease Control and Prevention. Coronavirus disease 2019 (COVID-19) (2020) prevention and treatment. Accessed on 24 May 2020. Available from: https://www.cdc.gov/coronavirus/ 2019-ncov/hcp/clinical-guidance-management-patients.html

5. National Institute for Health and Care Excellence (NICE 2020) COVID-19 rapid guideline: rheumatological autoimmune, inflammatory and metabolic bone disorders (NG167). Available from: https://www.nice.org.uk/guidance/ng167

6. British Society of Rheumatology (BSR) (2020) Guidance to rheumatologist. Available from: https://www.rheumatology.org.uk/ news-policy/details/Covid19-Coronavirus-update-members

7. British Society of Rheumatology (BSR) (2020) Risk stratification of patients with autoimmune rheumatic diseases. BSR 2020. Available from; https://www.nras.org.uk/data/files/COVID19 risk_scoring_guide.pdf. Accessed on 24 May 2020

8. Price E, MacPhie E, Kay L et al (2020) Identifying rheumatic disease patients at high risk and requiring shielding during the COVID-19 pandemic. Clin Med (Lond). Clinmed.2020-0160. https://doi.org/10.7861/clinmed.2020-0160

9. Favalli EG, Ingegnoli F, De Lucia O et al (2020) COVID-19 infection and rheumatoid arthritis: faraway, so close! Autoimmun Rev 19(5):102523. https://doi.org/10.1016/j.autrev.2020.102523
10. Bateman J, Mulherin D, Hirsch G, Venkatachalam S, Sheeran T (2020) Rapid distribution of information by SMS-embedded video link to patients during a pandemic. Lancet Rheumatol 2:e315-e316. https://doi.org/10.1016/S2665-9913(20)30126-0

11. Martinengo L, Spinazze P, Car J (2020) Mobile messaging with patients. BMJ. 2368:m884. https://doi.org/10.1136/bmj.m884

12. Greenhalgh T, Wherton J, Shaw $S$ et al (2020) Video consultations for COVID-19. BMJ 368:m998. https://doi.org/10.1136/bmj.m998

13. NHS England and NHS Improvement (2020) Attend Anywhere. Available from: https://england.nhs.attendanywhere.com/ resourcecentre/Content/Public Topics/Discover.htm (accessed 24 May 2020)

14. Finset A, Bosworth H, Butow P, Gulbrandsen P, Hulsman RL, Pieterse AH, Street R, Tschoetschel R, van Weert J (2020) Effective health communication - a key factor in fighting the COVID-19 pandemic. Patient Educ Couns 103(5):873-876. https://doi.org/10.1016/j.pec.2020.03.027

15. Haberman R, Axelrad J, Chen A et al (2020) Covid-19 in immunemediated inflammatory diseases - case series from New York. N Engl J Med NEJMc 2009567. https://doi.org/10.1056/ NEJMc2009567

16. Iyengar K, Jain VK, Vaishya R (2020) Pitfalls in telemedicine consultations in the era of COVID 19 and how to avoid them. Diabetes Metab Syndr 14(5):797-799. Advance online publication. https:// doi.org/10.1016/j.dsx.2020.06.007

17. Wood PR, Caplan L (2019) Outcomes, satisfaction, and costs of a rheumatology telemedicine program: a longitudinal evaluation. J Clin Rheumatol 25(1):41-44. https://doi.org/10.1097/RHU. 0000000000000778

Publisher's note Springer Nature remains neutral with regard to jurisdictional claims in published maps and institutional affiliations. 